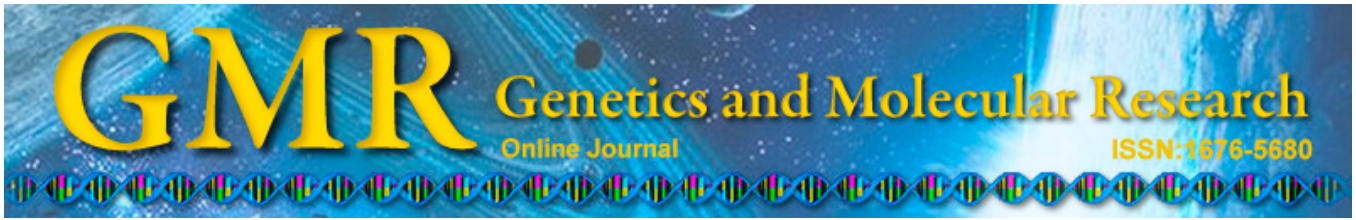

\title{
Functional polymorphisms in microRNA gene and hepatitis $B$ risk among Asian population: a meta-analysis
}

\author{
G.Q. Zhou, H. Meng, J.R. Wang, F.X. Sun, X.J. Wang, R.B. Wang and \\ X.B. Wang \\ Center of Integrated Traditional Chinese and Western Medicine, \\ Beijing Ditan Hospital Affiliated to Capital Medical University, Beijing, China \\ Corresponding author: G.Q. Zhou \\ E-mail: guiqin_zhou@126.com
}

Genet. Mol. Res. 14 (2): 4767-4777 (2015)

Received August 8, 2014

Accepted December 8, 2014

Published May 11, 2015

DOI http://dx.doi.org/10.4238/2015.May.11.9

\begin{abstract}
Genetic mutations in microRNA gene can alter expression, which may interact to increase the risk of developing various diseases, including hepatitis B. However, published results are inconclusive or ambiguous. The aim of this review and meta-analysis is to more precisely estimate the association between polymorphisms in microRNA genes and hepatitis B risk. A digital search was performed of the MEDLINE EMBASE, CNKI, and CBM databases to identify relevant articles published up to February 18, 2014. Ten case-control studies were included, with a total of 6042 patients with hepatitis B and 6834 healthy controls. Nine single-nucleotide polymorphisms in the miRNA gene were examined, including miR-34b/c [rs4938723 (T>C)], miR-196a-2 [rs11614913 (C>T)], miR-146a [rs2910164 (G>C)], miR-499 [rs3746444 (T>C)], miR-122 [rs3783553 (ins/del)], miR149 [rs2292832 (C>T)], miR-106b-25 [rs999885 (A>G)], miR-let-7c [rs6147150 (ins/del)], and miR-218 [rs11134527 (A>G)]. The metaanalysis results indicated that the miR-196a- $2 * \mathrm{~T}$, miR-122*del, miR$106 \mathrm{~b}-25 * \mathrm{~A}$, and miR-let- $7 \mathrm{c} *$ del alleles/carriers increase the risk of
\end{abstract}


hepatitis B among the Asian population. However, the miR-146a, miR499, miR-149, miR-218, and miR-34b/c polymorphisms may not be linked with the risk of hepatitis B. Further investigations are warranted to determine the exact associations between microRNA mutations and hepatitis B susceptibility.

Key words: Hepatitis B; Meta-analysis; MicroRNA; Polymorphism

\section{INTRODUCTION}

Hepatitis involves inflammation of the liver; there are at least 5 types of hepatitis, known as types A, B, C, D, and E, which are most commonly caused by viral infection. Hepatitis B virus (HBV), a hepatotropic DNA virus from the Hepadnaviridae family, is a major cause of liver diseases, including chronic hepatitis and liver cirrhosis (Ren et al., 2012). HBV can be transmitted vertically and horizontally through sexual or household contact with an infected person, perinatal transmission and nosocomial exposure, or by unsafe injections (Poovorawan et al., 2002; Shepard et al., 2006). Over 2 billion people are estimated to be infected with HBV and approximately 378 million have chronic infection; 1-2 million deaths occur annually from HBV-related liver disease or hepatocellular carcinoma (HCC) (Nath et al., 2010). Among the etiological factors thought to be associated with HCC development, such as carcinogen/toxin exposure and/or genetic factors, HBV infection accounts for more than $60 \%$ of the total $\mathrm{HCC}$ in developing countries and less than a quarter of cases in developed countries (Jemal et al., 2011). Specific miRNAs act as mediators of changes in the tumor microenvironment during HCC progression, and there may be a link between HBV statuses as etiological factors. Yang et al. (2012) found that HBV infection status and transforming growth factor-(TGF)- $\beta$ signaling activity are positively associated with portal vein tumor thrombus development in HCC patients.

Various gene mutations may be related to hepatitis (Sayan et al., 2011; Xin et al., 2011; Li et al., 2012; Lu et al., 2012), and 2 polymorphisms in microRNA, miR-196a-2 and miR-106b-25, have been found to be closely related to hepatitis B (Qi et al., 2010; Liu et al., 2012). Since its discovery, hundreds of single-nucleotide polymorphisms (SNPs) in microRNA have been reported and a mature regulatory network of microRNAs has been established (Ason et al., 2006; Berezikov et al., 2006; Kloosterman et al., 2006; Ruby et al., 2006). Most microRNA polymorphisms are generated as a stem-loop containing primary miRNA (pri-miRNA) (Cai et al., 2004; Lee et al., 2004a,b). After translation, the pri-miRNA is processed within the nucleus by RNase III enzyme, producing a $\sim 70$-nucleotide (nt) hairpin precursor miRNA (pre-miRNA) (Landthaler et al., 2004). The pre-miRNA is then cleaved to produce the mature $\sim 22$-nt miRNA by Dicer (Forstemann et al., 2005). Subsequently, Ago2, together with Dicer, form the RNA-induced silencing complex and cleave and degrade the target mRNA by guiding it into the RNA-induced silencing complex (Du and Zamore, 2005). Recently, several studies indicated that serum microRNA-122 levels alter the cell cycle and thus serve as biomarkers for HBV infections (Fehr et al., 2012; Waidmann et al., 2012; Wang et al., 2012). Potenza et al. (2011) also demonstrated that hsa-miR-125a-5p interacts with HBV sequences and represses HBV surface antigen (HBsAg) expression. In addition, hsamiR-125a-5p and $-151-5 p$ regulate HBV replication in humans by altering TGF signaling (Park et al., 2012). Interestingly, HBV may in turn alter the microRNA expression pattern by exerting a negative influence on the microRNA processor Drosha (Ren et al., 2012). Studies have also 
shown that miR-338-3p is down regulated by HBV X and inhibits cell proliferation by targeting the 3'-untranslated region of cyclinD1 (Fu et al., 2012).

Based on these observations, we hypothesized that microRNA genes may be closely related to hepatitis B. However, the regulatory mechanisms are not consistent among studies, and individually published data focusing on the associations are inconclusive or ambiguous (Gao et al., 2009; Akkiz et al., 2011; Kim et al., 2012; Yu et al., 2012; Han et al., 2013b). Thus, we conducted the present meta-analysis to determine the association between miRNA gene polymorphisms and the risk of hepatitis B. To our knowledge, this is the first meta-analysis to assess the relationship between polymorphisms in microRNA genes and hepatitis B susceptibility.

\section{MATERIAL AND METHODS}

The present meta-analysis was conducted according to the guidelines of the Preferred Reporting Items for Systematic Reviews and Meta-analyses.

\section{Literature search}

Relevant papers published before February 18, 2014 were identified through a search of the MEDLINE EMBASE, CNKI, and CBM databases using the following terms: ("microRNAs" or "miRNAs" or "primary microRNA" or "primary miRNA" or "pri-miRNA" or "small temporal RNA" or "pre-miRNA") and ("hepatitis B" or "hepatitis B, chronic" or "chronic hepatitis B" or "hepatitis B virus" or "B virus, hepatitis") and ("polymorphism" or "SNP" or "gene mutation" or "genetic variants"). The references in eligible articles and textbooks were also reviewed to identify other potential studies. Any disagreements were resolved through discussion between the respective authors.

\section{Inclusion and exclusion criteria}

Studies included in our meta-analysis met the following criteria: a) case-control or cohort studies focused on the associations between microRNA polymorphisms and hepatitis B risk; b) all patients diagnosed with hepatitis B were consistently positive for HBsAg and were diagnosed clinically; c) published data regarding the allele and genotype frequencies of SNPs must meet sufficient requirements. Studies were excluded when they were: a) not case-control or cohort studies; b) duplicates or continued studies of previous publications; c) based on incomplete data; and d) other meta-analyses, letters, reviews, and editorial articles. If more than one study by the same author using the same case series was published, either the study with the larger sample size or the most recently published study was selected.

\section{Data extraction}

Using a standardized form, data from studies included were extracted independently by 2 authors. For each study, the following characteristics were collected: first author, year of publication, country of origin, number of cases and controls, mean age, and gender ratio, genotype method, allele and genotype frequencies, and evidence of Hardy-Weinberg equilibrium (HWE) in controls. In cases of conflicting evaluations, 2 authors reached a consensus through discussion; if no agreement could be reached, a third author made the decision. 


\section{Quality assessment of studies included}

Two authors independently assessed the quality of the articles published according to Newcastle-Ottawa Scale (NOS) criteria (Supplementary file) (Stang, 2010). Nine assessment items matching the quality appraisals were used in this meta-analysis, with scores ranging from 0-9. A score of more than 5 stars indicated moderate to high quality. The 2 authors resolved differences through discussion.

\section{Statistical analysis}

The strength of the associations between the microRNA polymorphisms and hepatitis B risk were measured using crude odds ratios (ORs) with their corresponding 95\% confidence intervals (CIs) using 5 genetic models, including allele ( $\mathrm{M}$ allele $v s \mathrm{~W}$ allele), dominant ( $\mathrm{MM}+\mathrm{MW}$ $v s \mathrm{WW}$ ), recessive (MM vs WW $+\mathrm{MW})$, homozygous (MM vs WW), and heterozygous (MM vs WM) models. The statistical significance of the pooled ORs was examined using the $Z$ test. Between-study variations and heterogeneities were estimated using Cochran's $Q$-statistic with a $\mathrm{P}<$ 0.05 as a cutoff for statistically significant heterogeneity (Higgins and Thompson, 2002). We also quantified the effect of heterogeneity using the $I^{2}$ test (ranges from $0-100 \%$ ), which represents the proportion of inter-study variability that can be attributed to heterogeneity rather than to chance (Zintzaras and Ioannidis, 2005). When the heterogeneity was absent $(\mathrm{P}>0.1)$ and $I^{2}<50 \%$, the fixed-effects model (the Mantel-Haenszel method) was used to estimate the summarized OR; otherwise, the random-effects model (the DerSimonian-Laird method) was used for meta-analysis. To establish the effects of heterogeneity based on the results of the meta-analyses, we also tested whether the genotype frequencies of controls were in HWE using the $\chi^{2}$ test. To ensure the reliability of the results, sensitivity analysis was performed by omitting individual studies. Begg's funnel plots were used to detect publication bias. Egger's linear regression test, a linear regression approach to measure funnel plot asymmetry on the natural logarithm scale of the OR, was also used to evaluate publication bias (Peters et al., 2006). All P-values were 2-sided. Analyses were calculated using the STATA Version 12.0 software (Stata Corp, College Station, TX, USA).

\section{RESULTS}

\section{Characteristics of included studies}

A total of 104 potentially relevant articles were identified; after removing duplicates, 94 articles were excluded, and 10 eligible studies including 6042 patients with hepatitis B and 6834 healthy controls met our inclusion criteria for this meta-analysis (Gao et al., 2009; Qi et al., 2010; Akkiz et al., 2011; Zhang, 2011; Kim et al., 2012; Liu et al., 2012; Xiang et al., 2012; Yu et al., 2012; Han et al., 2013a,b). The details of the selection process are presented in a flow chart in Figure 1. The publication years of the 10 studies were $2009(\mathrm{~N}=1), 2010(\mathrm{~N}=$ 1), $2011(\mathrm{~N}=2), 2012(\mathrm{~N}=4)$, and $2013(\mathrm{~N}=2)$. All patients diagnosed with hepatitis B were consistently positive for HBsAg and were diagnosed clinically. The genotype distributions of each SNP in controls were accessed for HWE, and all studies included were in accordance with HWE except for 2 studies $(\mathrm{P}<0.05)$ (Gao et al., 2009; Liu et al., 2012). According to the Newcastle-Ottawa Scale quality score system, the scores of all included studies were higher than 5 points. Figure 2 summarizes the review of authors' judgments regarding each item 
presented as percentages across studies included. Main characteristics and methodological qualities of all eligible studies are listed in Table 1.

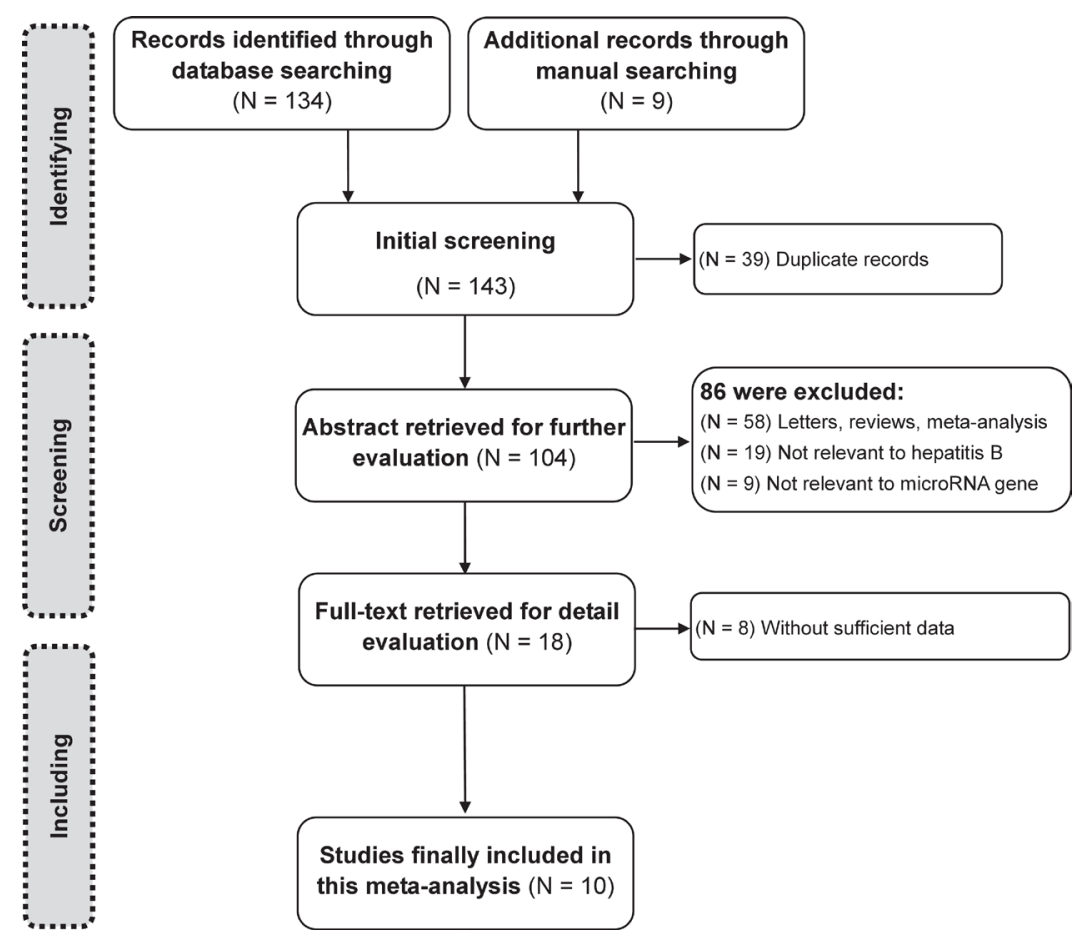

Figure 1. Flow diagram of selection of studies and specific reasons for exclusion from the present meta-analysis.

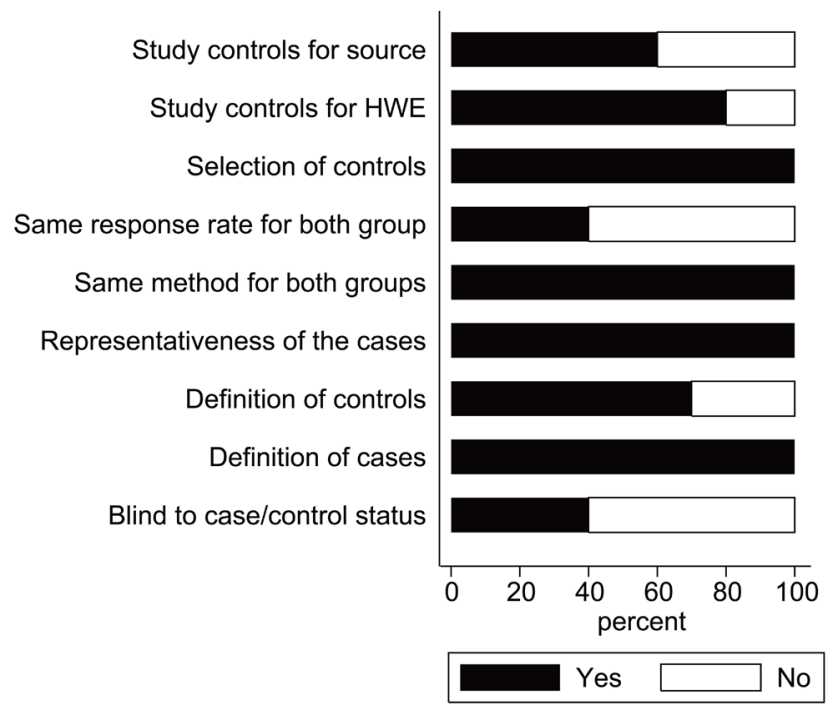

Figure 2. NOS quality assessment scale for studies included in this meta-analysis. 


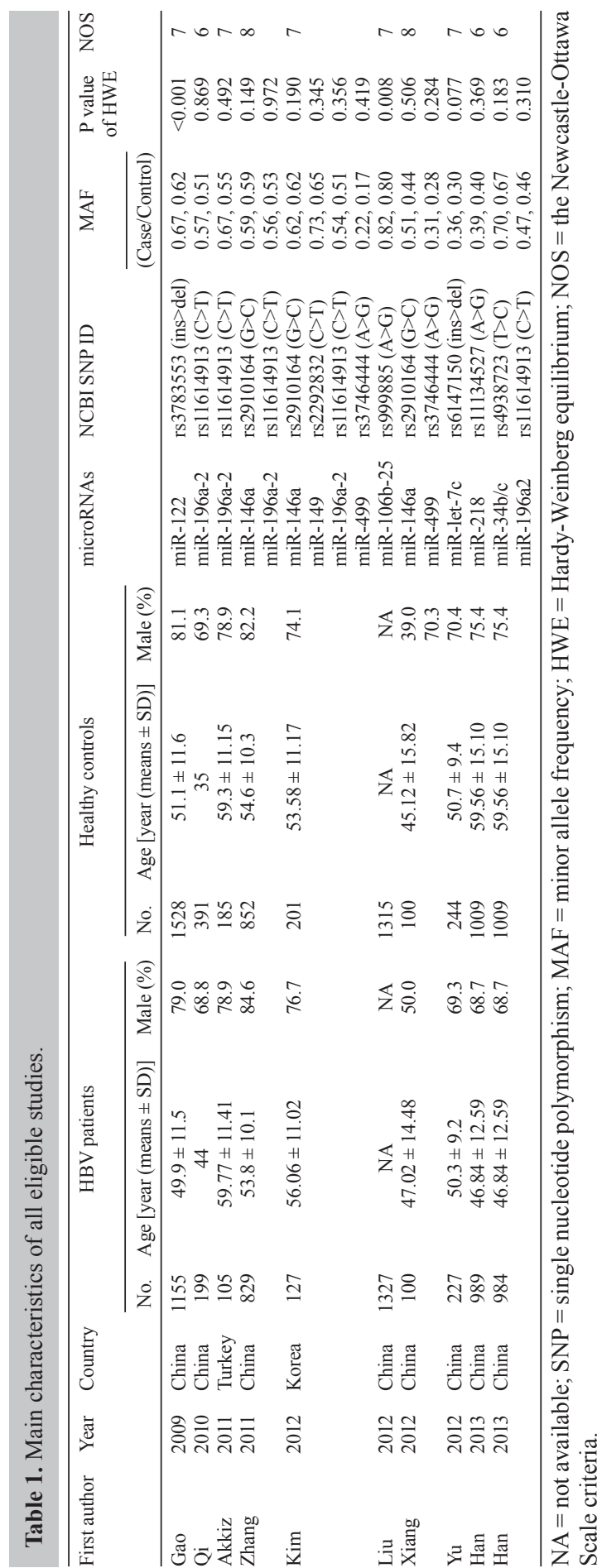




\section{Quantitative data synthesis}

A total of 9 SNPs in microRNA were examined, including miR-196a-2, miR-146a, miR-499, miR-122, miR-149, miR-106b-25, miR-let-7c, and miR-218. A quantitative summary on the findings regarding the association between the microRNA polymorphisms and risk of hepatitis B is shown in Table 2. Overall, there appears to be an increased risk of hepatitis $\mathrm{B}$ associated with the microRNA substitution under 4 genetic models (allele: $\mathrm{OR}=1.13$, 95\%CI: $1.08-1.17, \mathrm{P}=0.006$; dominant: $\mathrm{OR}=1.13,95 \% \mathrm{CI}: 1.04-1.22, \mathrm{P}=0.003$; recessive: $\mathrm{OR}=1.19,95 \% \mathrm{CI}: 1.11-1.27, \mathrm{P}<0.001$; homozygous: $\mathrm{OR}=1.21,95 \% \mathrm{CI}: 1.09-1.33, \mathrm{P}<$ 0.001) (Figure 3). For the miR-196a-2 polymorphism, 5 studies were included. The results showed that miR-196a-2*T allele/carriers may have an increased risk of hepatitis B (allele: $\mathrm{OR}=1.11,95 \% \mathrm{CI}: 1.03-1.21, \mathrm{P}=0.010$; dominant: $\mathrm{OR}=1.18,95 \% \mathrm{CI}: 1.03-1.35, \mathrm{P}=0.018$; homozygous: $\mathrm{OR}=1.25,95 \% \mathrm{CI}: 1.06-1.47, \mathrm{P}=0.008)$. Similar association were observed between hepatitis $\mathrm{B}$ and polymorphisms in miR-122 (allele: $\mathrm{OR}=1.23,95 \% \mathrm{CI}: 1.10-1.38, \mathrm{P}<$ 0.001 ; recessive: $\mathrm{OR}=1.41,95 \% \mathrm{CI}: 1.21-1.65, \mathrm{P}<0.001$; homozygous: $\mathrm{OR}=1.38,95 \% \mathrm{CI}$ : 1.07-1.79, $\mathrm{P}=0.013$; heterozygous: $\mathrm{OR}=1.42,95 \% \mathrm{CI}: 1.21-1.67, \mathrm{P}<0.001)$, miR-106b-25 (allele: $\mathrm{OR}=1.18,95 \% \mathrm{CI}: 1.02-1.35, \mathrm{P}=0.021$; recessive: $\mathrm{OR}=1.27,95 \% \mathrm{CI}: 1.08-1.49, \mathrm{P}$ $=0.004$; heterozygous: $\mathrm{OR}=1.31,95 \% \mathrm{CI}: 1.11-1.54, \mathrm{P}=0.002$ ), and miR-let-7c (allele: OR $=1.35,95 \%$ CI: $1.03-1.77, \mathrm{P}=0.031$; dominant: $\mathrm{OR}=1.48,95 \% \mathrm{CI}: 1.03-2.13, \mathrm{P}=0.034$ ). However, for the miR-146a, miR-499, miR-149, miR-218, and miR-34b/c polymorphisms, our meta-analysis results showed that these polymorphisms may not be linked with the risk of hepatitis $\mathrm{B}$ under any genetic models (all $\mathrm{P}>0.05)$.

\begin{tabular}{|c|c|c|c|c|c|c|c|c|c|c|}
\hline \multirow[t]{2}{*}{ miRNA genes } & \multicolumn{2}{|c|}{$\begin{array}{l}\mathrm{M} \text { allele } v s \mathrm{~W} \text { allele } \\
\text { (Allele model) }\end{array}$} & \multicolumn{2}{|c|}{$\begin{array}{l}\mathrm{MM}+\mathrm{MW} v s \mathrm{WW} \\
\text { (Dominant model) }\end{array}$} & \multicolumn{2}{|c|}{$\begin{array}{l}\text { MM } v s \text { WW + MW } \\
\text { (Recessive model) }\end{array}$} & \multicolumn{2}{|c|}{$\begin{array}{c}\text { MM vs WW } \\
\text { (Homozygous model) }\end{array}$} & \multicolumn{2}{|c|}{$\begin{array}{c}\text { MM } v s \text { WM } \\
\text { (Heterozygous model) }\end{array}$} \\
\hline & OR $(95 \% \mathrm{CI})$ & $\mathrm{P}$ value & OR $(95 \% \mathrm{CI})$ & $P$ value & OR $(95 \% \mathrm{CI})$ & $\mathrm{P}$ value & OR $(95 \% \mathrm{CI})$ & $P$ value & OR $(95 \% \mathrm{CI})$ & $P$ value \\
\hline miR-196a-2 $(C>T)$ & $1.11(1.03-1.21)$ & 0.010 & $1.18(1.03-1.35)$ & 0.018 & $1.13(0.99-1.28)$ & 0.065 & $1.25(1.06-1.47)$ & 0.008 & $1.08(0.94-1.24)$ & 0.290 \\
\hline $\operatorname{miR}-146 a(G>C)$ & $1.02(0.90-1.15)$ & 0.762 & $1.06(0.85-1.32)$ & 0.618 & $1.00(0.84-1.20)$ & 0.967 & $1.04(0.81-1.34)$ & 0.744 & $0.99(0.82-1.20)$ & 0.945 \\
\hline miR-499a $(\mathrm{T}>\mathrm{C})$ & $1.28(0.96-1.69)$ & 0.092 & $1.31(0.93-1.85)$ & 0.123 & $1.49(0.71-3.10)$ & 0.288 & $1.56(0.73-3.90)$ & 0.248 & $1.38(0.63-3.03)$ & 0.418 \\
\hline miR-122 (Ins>Del) & $1.23(1.10-1.38)$ & $<0.001$ & $1.15(0.90-1.46)$ & 0.271 & $1.41(1.21-1.65)$ & $<0.001$ & $1.38(1.07-1.79)$ & 0.013 & $1.42(1.21-1.67)$ & $<0.001$ \\
\hline miR-149 $(\mathrm{C}>\mathrm{T})$ & $1.42(1.00-2.00)$ & 0.047 & $1.36(0.62-3.00)$ & 0.439 & $1.64(1.05-2.56)$ & 0.052 & $1.72(0.76-3.90)$ & 0.194 & $1.62(1.01-2.59)$ & 0.051 \\
\hline miR-106b-25 (G>A) & $1.18(1.02-1.35)$ & 0.021 & $0.87(0.56-1.35)$ & 0.528 & $1.27(1.08-1.49)$ & 0.004 & $0.95(0.61-1.48)$ & 0.808 & $1.31(1.11-1.54)$ & 0.002 \\
\hline miR-let-7c $($ Ins $>$ Del $)$ & $1.35(1.03-1.77)$ & 0.031 & $1.48(1.03-2.13)$ & 0.034 & $1.37(0.79-2.35)$ & 0.260 & $1.62(0.91-2.87)$ & 0.101 & $1.12(0.63-2.01)$ & 0.698 \\
\hline $\operatorname{miR}-218(\mathrm{~A}>\mathrm{G})$ & $0.98(0.87-1.12)$ & 0.818 & $0.95(0.79-1.14)$ & 0.595 & $1.03(0.82-1.30)$ & 0.794 & $0.99(0.77-1.29)$ & 0.971 & $1.06(0.83-1.36)$ & 0.635 \\
\hline $\mathrm{miR}-34 \mathrm{~b} / \mathrm{c}(\mathrm{C}>\mathrm{T})$ & $1.14(0.99-1.30)$ & 0.058 & $1.28(0.96-1.70)$ & 0.090 & $1.14(0.96-1.36)$ & 0.149 & $1.33(0.99-1.80)$ & 0.058 & $1.09(0.91-1.32)$ & 0.348 \\
\hline Overall & $1.13(1.08-1.17)$ & 0.006 & $1.13(1.04-1.22)$ & 0.003 & $1.19(1.11-1.27)$ & $<0.001$ & $1.21(1.09-1.33)$ & $<0.001$ & $1.17(1.09-1.26)$ & $<0.001$ \\
\hline
\end{tabular}

$\mathrm{W}=$ wild allele; $\mathrm{M}=$ mutant allele; $\mathrm{OR}=$ odd ratio; $\mathrm{CI}=$ confidence interval.

\section{Sensitivity analysis and publication bias}

Sensitivity analyses were performed to assess the influence of individual studies on pooled ORs by omitting individual studies. The results suggested that no individual study significantly affected the pooled ORs of microRNA polymorphisms under the allele model, indicating that our results are statistically robust. Begg's funnel plot and Egger's linear regression test were performed on the meta-data to assess publication bias of the individual studies. The shapes of the funnel plots of microRNA polymorphisms did revealed no evidence of obvious 
asymmetry. Egger's test also showed no significant statistical evidence of publication in the allele model $(t=-0.60, \mathrm{P}=0.557)$ (Figure 4).

A

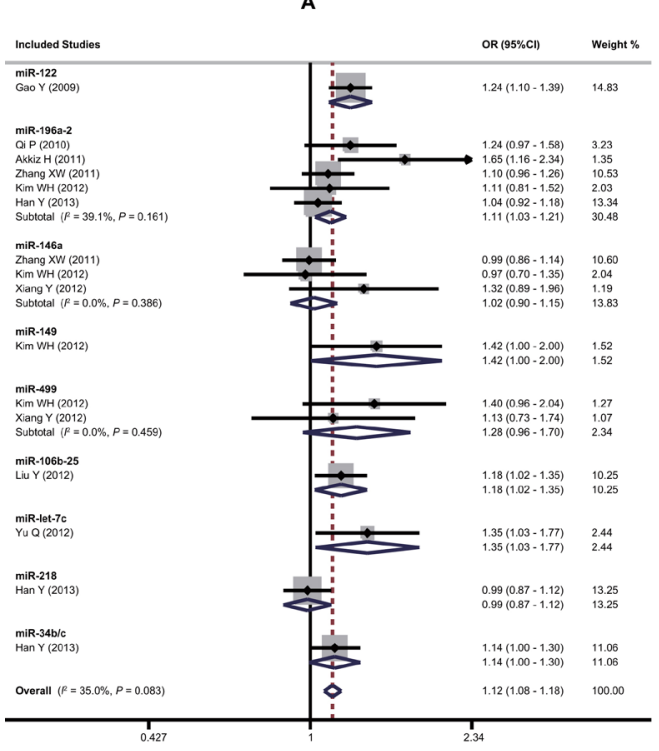

B

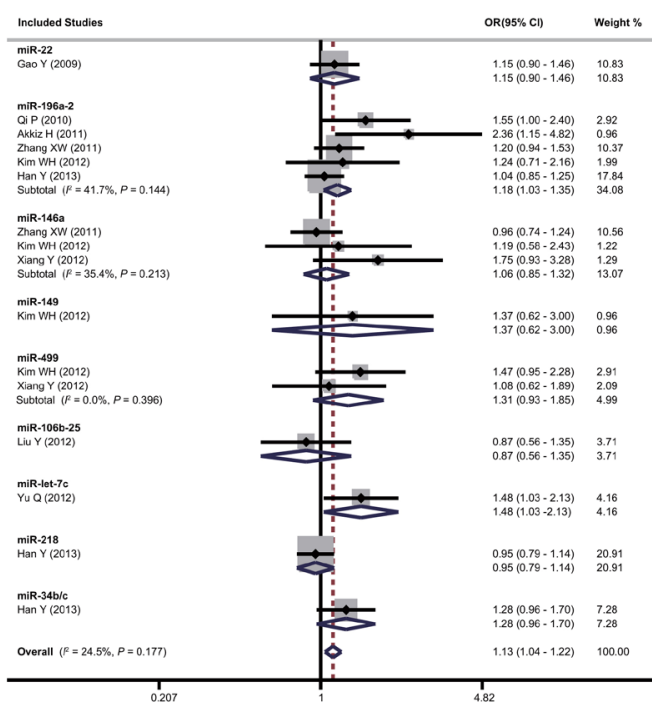

Figure 3. Forest plot of ORs for the association between microRNA polymorphisms and hepatitis B risk illustrated under the allele (A) and dominant (B) models.

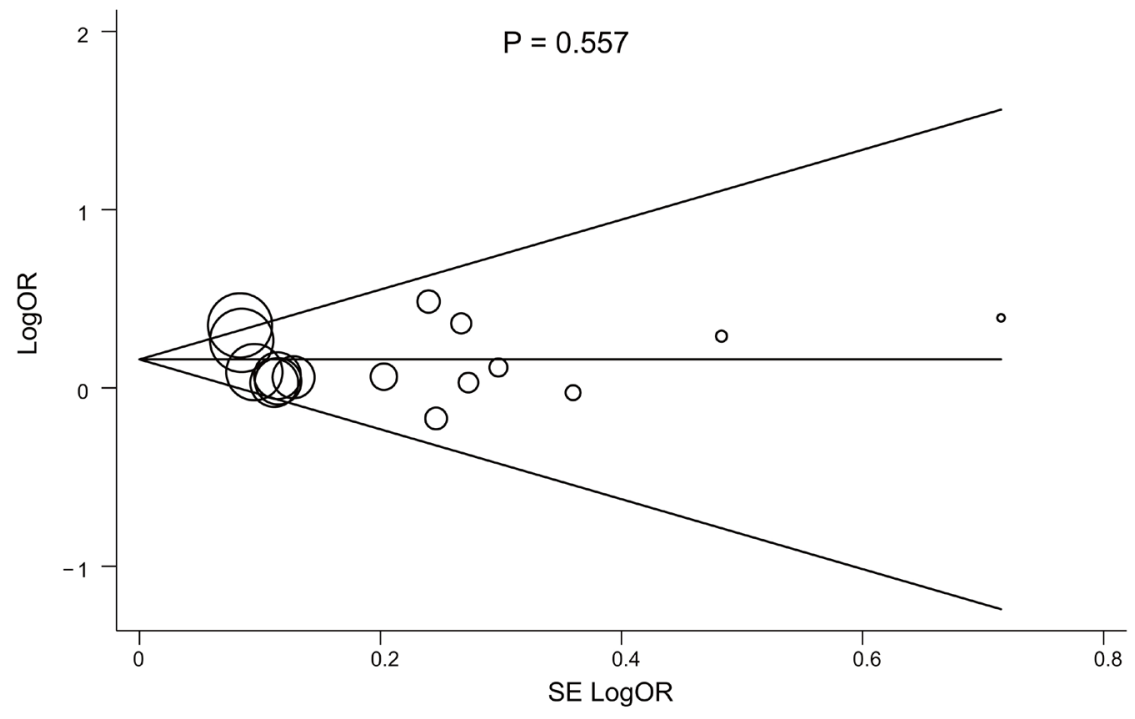

Figure 4. Begg's funnel plot of publication bias in study selection on microRNA polymorphisms are illustrated under the allele model. Each point represents a separate study by the indicated association. $\log [\mathrm{OR}]$, natural logarithm of OR. Horizontal line, mean magnitude of the effect. 


\section{DISCUSSION}

Currently, numerous studies have been conducted to determine the role of SNPs present in precursor and mature microRNAs and their influences on susceptibility and progression of various diseases (Xu et al., 2011). Not all SNPs located in the regions of microRNA affect miRNA expression, but some play a dominant role in modulating target mRNAs (Potenza et al., 2011; Fu et al., 2012). Hepatitis B is a disease caused by HBV infection, which is a major cause of liver diseases, including chronic hepatitis and liver cirrhosis (Ren et al., 2012). In addition to vertical and horizontal transmission, other factors may contribute to hepatitis B, such as mutations in microRNA (Gao et al., 2011; Lu et al., 2012), both of which are currently widely studied. The correlations between various SNPs present in microRNA and hepatitis B susceptibility have been widely studied. Gao et al. (2011) found that miRNA deregulation occurred in HBV-associated HCC development and accumulated with the various stages of hepatocarcinogenesis, which may drive disease progression. Several studies have shown that among chronic carriers and liver cirrhosis patients, the A allele of rs3859501 and the haplotype pri-miRNAs-371-373 ht2 were more protective against HCC than other genotypes and haplotypes (Kwak et al., 2012).

These studies used relatively small or sometimes inconsistent sample sizes. Thus, these studies may not have been sufficiently powerful to detect minor effects of microRNA polymorphisms on hepatitis B. In this meta-analysis, we attempted to increase the understanding of the relationships between microRNA polymorphisms and hepatitis B risk. Several genetic variants targeted by miR-122, miR-196a-2, miR-146a, miR-106b-25, miR-499, and miR-let-7c have been reported to affect the strength of microRNA binding, affecting the regulation of target genes and individual susceptibility to hepatitis B (Gao et al., 2009; Qi et al., 2010; Liu et al., 2012; Xiang et al., 2012). In accordance with previous studies, the result of our meta-analysis of 10 independent studies showed significant associations between polymorphisms in miR-196a-2, miR-122, miR-106b-25, and miR-let-7c and altered hepatitis B risk, indicating an integral role of microRNA in the diverse processes of hepatitis $\mathrm{B}$, including the immune response and tumorigenesis. Mutations in microRNA can affect not only the patterns of interactions between hepatitis B virus and the host immune system, but also various phases of malignant processes, such as carcinogenesis, tumor growth, and invasiveness; our findings indicate that microRNA is a promising target for immunotherapy, early diagnosis, and intervention of hepatitis B. However, we observed no significant correlations between miR146a, miR-499, miR-149, miR-218, and miR-34b/c and increased hepatitis B susceptibility. Similarly, a recent study was unable to support a significant contribution to hepatitis risk by the miR-146a mutation, while a significant difference was observed in ORs of the miR-499 $\mathrm{C} / \mathrm{C}$ genotype compared with individuals the carrying TT genotype, suggesting higher susceptibility to hepatitis B (Xiang et al., 2012). The reason behind this discrepancy may be because different populations in different areas and relatively small sample sizes were included in our meta-analysis. Moreover, the extremely rare level and low frequency of miR-499, miR-149, miR-218, and miR-34b/c mutations in hepatitis B patients may make it difficult to identify nucleotide changes in corresponding genes. Therefore, genetic polymorphisms in microRNA should be considered as related to hepatitis B susceptibility because some functionally-deficient variants of microRNA may also act as low-penetrance risk factors for hepatitis B.

There were some limitations to the present study. First, all participants in the studies included in this meta-analysis were Asian; although the correlations were strong for Asians, 
additional studies in Caucasian and African populations are required. Second, the number of subjects included in the meta-analysis was too small to perform subgroup analysis for each polymorphism. Additionally, lack of available information prevented more precise evaluation with adjusted ORs using specific covariates such as age and smoking status, making our data conservative estimates of the association between microRNA polymorphisms and risk of HBV infection. Because of these limitations, our results should be confirmed in further studies. However, this is the first meta-analysis to investigate the correlation between microRNA polymorphisms and hepatitis B risk.

In conclusion, in this meta-analysis, we found that miR-196a-2*T allele, miR-122* del, miR-106b-25*A, and miR-let-7c*del alleles/carriers may increase the risk of hepatitis B among the Asian population, while the miR-146a, miR-499a, miR-149, miR-218, and miR$34 \mathrm{~b} / \mathrm{c}$ polymorphisms may not be risk factors for hepatitis $\mathrm{B}$. These findings provide novel insights into the biological mechanism of hepatitis B. These relationships may provide a more comprehensive understanding of how microRNA mutations function in hepatitis and will benefit the design of therapy targets for corresponding treatments. Therefore, further investigations are warranted to reveal the exact associations between various microRNA mutations and hepatitis B susceptibility.

\section{Supplementary material}

\section{REFERENCES}

Akkiz H, Bayram S, Bekar A, Akgollu E, et al. (2011). A functional polymorphism in pre-microRNA-196a-2 contributes to the susceptibility of hepatocellular carcinoma in a Turkish population: a case-control study. J. Viral Hepat. 18: e399-e407.

Ason B, Darnell DK, Wittbrodt B, Berezikov E, et al. (2006). Differences in vertebrate microRNA expression. Proc. Natl. Acad. Sci. U. S. A. 103: 14385-14389.

Berezikov E, Cuppen E and Plasterk RH (2006). Approaches to microRNA discovery. Nat. Genet. 38 (Suppl): S2-S7.

Cai X, Hagedorn CH and Cullen BR (2004). Human microRNAs are processed from capped, polyadenylated transcripts that can also function as mRNAs. RNA 10: 1957-1966.

Du T and Zamore PD (2005). microPrimer: the biogenesis and function of microRNA. Development 132: 4645-4652.

Fehr C, Conrad KD and Niepmann M (2012). Differential stimulation of hepatitis C virus RNA translation by microRNA-122 in different cell cycle phases. Cell Cycle 11: 277-285.

Forstemann K, Tomari Y, Du T, Vagin VV, et al. (2005). Normal microRNA maturation and germ-line stem cell maintenance requires Loquacious, a double-stranded RNA-binding domain protein. PLoS Biol. 3: e236.

Fu X, Tan D, Hou Z, Hu Z, et al. (2012). miR-338-3p is downregulated by hepatitis B virus X and inhibits cell proliferation by targeting the 3'-UTR tegion of cyclinD1. Int. J. Mol. Sci. 13: 8514-8539.

Gao P, Wong CC, Tung EK, Lee JM, et al. (2011). Deregulation of microRNA expression occurs early and accumulates in early stages of HBV-associated multistep hepatocarcinogenesis. J. Hepatol. 54: 1177-1184.

Gao Y, He Y, Ding J, Wu K, et al. (2009). An insertion/deletion polymorphism at miRNA-122-binding site in the interleukin-1alpha 3' untranslated region confers risk for hepatocellular carcinoma. Carcinogenesis 30: 2064-2069.

Han Y, Pu R, Han X, Zhao J, et al. (2013a). Association of a potential functional pre-miR-218 polymorphism and its interaction with hepatitis B virus mutations with hepatocellular carcinoma risk. Liver Int. 34: 728-736.

Han Y, Pu R, Han X, Zhao J, et al. (2013b). Associations of pri-miR-34b/c and pre-miR-196a2 polymorphisms and their multiplicative interactions with hepatitis B virus mutations with hepatocellular carcinoma risk. PLoS One 8: e58564.

Higgins JP and Thompson SG (2002). Quantifying heterogeneity in a meta-analysis. Stat. Med. 21: 1539-1558.

Jemal A, Bray F, Center MM, Ferlay J, et al. (2011). Global cancer statistics. CA Cancer J. Clin. 61: 69-90.

Kim WH, Min KT, Jeon YJ, Kwon CI, et al. (2012). Association study of microRNA polymorphisms with hepatocellular carcinoma in Korean population. Gene 504: 92-97.

Kloosterman WP, Steiner FA, Berezikov E, de Bruijn E, et al. (2006). Cloning and expression of new microRNAs from zebrafish. Nucleic Acids Res. 34: 2558-2569. 
Kwak MS, Lee DH, Cho Y, Cho EJ, et al. (2012). Association of polymorphism in pri-microRNAs-371-372-373 with the occurrence of hepatocellular carcinoma in hepatitis B virus infected patients. PLoS One 7: e41983.

Landthaler M, Yalcin A and Tuschl T (2004). The human DiGeorge syndrome critical region gene 8 and Its D. melanogaster homolog are required for miRNA biogenesis. Curr. Biol. 14: 2162-2167.

Lee Y, Kim M, Han J, Yeom KH, et al. (2004a). MicroRNA genes are transcribed by RNA polymerase II. EMBO J. 23: 4051-4060.

Lee YS, Nakahara K, Pham JW, Kim K, et al. (2004b). Distinct roles for Drosophila Dicer-1 and Dicer-2 in the siRNA/ miRNA silencing pathways. Cell 117: 69-81.

Li SH, Zhao H, Ren YY, Liu YZ, et al. (2012). The H63D mutation of the hemochromatosis gene is associated with sustained virological response in chronic hepatitis $\mathrm{C}$ patients treated with interferon-based therapy: a meta-analysis. Tohoku J. Exp. Med. 226: 293-299.

Liu Y, Zhang Y, Wen J, Liu L, et al. (2012). A genetic variant in the promoter region of miR-106b-25 cluster and risk of HBV infection and hepatocellular carcinoma. PLoS One 7: e32230.

Lu JJ, Chen EQ, Yang JH, Zhou TY, et al. (2012). A mutation in the interferon regulatory element of HBV may influence the response of interferon treatment in chronic hepatitis B patients. Virol J. 9: 10.

Nath A, Agarwal R, Malhotra P and Varma S (2010). Prevalence of hepatitis B virus infection in non-Hodgkin lymphoma: a systematic review and meta-analysis. Intern. Med. J. 40: 633-641.

Park SO, Kumar M and Gupta S (2012). TGF-beta and iron differently alter HBV replication in human hepatocytes through TGF-beta/BMP signaling and cellular microRNA expression. PLoS One 7: e39276.

Peters JL, Sutton AJ, Jones DR, Abrams KR, et al. (2006). Comparison of two methods to detect publication bias in metaanalysis. JAMA 295: 676-680.

Poovorawan Y, Chatchatee P, Chongsrisawat V (2002). Epidemiology and prophylaxis of viral hepatitis: a global perspective. J. Gastroenterol. Hepatol. 17 (Suppl): S155-S166.

Potenza N, Papa U, Mosca N, Zerbini F, et al. (2011). Human microRNA hsa-miR-125a-5p interferes with expression of hepatitis B virus surface antigen. Nucleic Acids Res. 39: 5157-5163.

Qi P, Dou TH, Geng L, Zhou FG, et al. (2010). Association of a variant in MIR 196A2 with susceptibility to hepatocellular carcinoma in male Chinese patients with chronic hepatitis B virus infection. Hum. Immunol. 71: 621-626.

Ren M, Qin D, Li K, Qu J, et al. (2012). Correlation between hepatitis B virus protein and microRNA processor Drosha in cells expressing HBV. Antiviral Res. 94: 225-231.

Ruby JG, Jan C, Player C, Axtell MJ, et al. (2006). Large-scale sequencing reveals 21U-RNAs and additional microRNAs and endogenous siRNAs in C. elegans. Cell 127: 1193-1207.

Sayan M, Akhan SC and Senturk O (2011). Frequency and mutation patterns of resistance in patients with chronic hepatitis B infection treated with nucleos(t)ide analogs in add-on and switch strategies. Hepat. Mon. 11: 835-842.

Shepard CW, Simard EP, Finelli L, Fiore AE, et al. (2006). Hepatitis B virus infection: epidemiology and vaccination. Epidemiol. Rev. 28: 112-125.

Stang A (2010). Critical evaluation of the Newcastle-Ottawa scale for the assessment of the quality of nonrandomized studies in meta-analyses. Eur. J. Epidemiol. 25: 603-605.

Waidmann O, Bihrer V, Pleli T, Farnik H, et al. (2012). Serum microRNA-122 levels in different groups of patients with chronic hepatitis B virus infection. J. Viral Hepat. 19: e58-e65.

Wang S, Qiu L, Yan X, Jin W, et al. (2012). Loss of microRNA 122 expression in patients with hepatitis B enhances hepatitis B virus replication through cyclin G(1)-modulated P53 activity. Hepatology 55: 730-741.

Xiang Y, Fan S, Cao J, Huang S, et al. (2012). Association of the microRNA-499 variants with susceptibility to hepatocellular carcinoma in a Chinese population. Mol. Biol. Rep. 39: 7019-7023.

Xin YN, Lin ZH and Xuan SY (2011). Are there more factors other than hepatitis B virus DNA level and A1762T/ G1764A mutation in liver tissue that also independently predict postoperative survival in hepatocellular carcinoma? Hepatology 54: 378.

Xu W, Xu J, Liu S, Chen B, et al. (2011). Effects of common polymorphisms rs11614913 in miR-196a2 and rs2910164 in miR-146a on cancer susceptibility: a meta-analysis. PLoS One 6: e20471.

Yang P, Li QJ, Feng Y, Zhang Y, et al. (2012). TGF-beta-miR-34a-CCL22 signaling-induced Treg cell recruitment promotes venous metastases of HBV-positive hepatocellular carcinoma. Cancer Cell 22: 291-303.

Yu Q, Zhou CX, Chen NS, Zheng SD, et al. (2012). A polymorphism within ErbB4 is associated with risk for hepatocellular carcinoma in Chinese population. World J. Gastroenterol. 18: 383-387.

Zhang XW (2011). Relationship between genetic polymorphism in microRNAs precursor and genetic predisposition of hepatocellular carcinoma. Nanjing Medical University.

Zintzaras E and Ioannidis JP (2005). Heterogeneity testing in meta-analysis of genome searches. Genet. Epidemiol. 28: 123-137. 